\title{
A theoretical multimodal assignment model for simulating intermodal trips
}

\author{
L. D’Acierno ${ }^{1}$, M. Gallo ${ }^{2}$ \& B. Montella ${ }^{1}$ \\ ${ }^{I}$ Department of Transportation Engineering, \\ 'Federico II' University of Naples, Italy \\ ${ }^{2}$ Department of Engineering, University of Sannio (Benevento), Italy
}

\begin{abstract}
This paper proposes a hyper-network model for simulating intermodal trips (such as park-and-ride trips) assuming elastic travel demand as regards mode choice. In particular, user flows on both road and transit networks can be estimated by the proposed model which is based on dummy links, with suitable cost functions, which are able to simulate virtually 'on-network' modal split and transfer operations. In particular, we state that, with these suitable functions, mathematical equivalence is ensured between the results obtained on the hypernetwork and those obtained by applying a general assignment model with elastic demand at mode choice level.

Keywords: hyper-network approach, elastic demand, cross nested logit, parkand-ride trips.
\end{abstract}

\section{Introduction}

The growth of urban traffic in larger cities and in metropolitan areas has produced in recent decades high congestion levels, mainly due to the preference of users for private cars. In these contexts, commuting from suburban zones to city centres has assumed very significant levels, with prominent interchange flows.

In order to reduce congestion in urban centres, in several cities park-and-ride facilities are located on the main access roads, for intercepting interchange flows; these facilities allow users to park their cars and continue the trip by transit systems (rapid rail transit, bus lines, etc.). The park-and-ride facilities are also known as mode interchange nodes. 
The main feature of a park-and-ride trip is that it is intermodal, in the sense that it is not univocally classifiable as road or transit: part of the trip is performed by private car and the other part by transit system. This feature also generates some theoretical and practical difficulties in simulating the system with usual mode choice and route choice models.

In this paper we propose a hyper-network approach for simulating jointly mode choice and route choice on a multimodal system where the available modes are road, transit system and park-and-ride (combined road-transit trips). The proposed approach can be seen as a method for solving the elastic demand assignment model (Cascetta [1]), assuming that only mode choice is elastic.

Several authors have proposed models that simulate jointly more than one travel choice; Tomlin [2], Florian et al. [3] and Evans [4] developed models for estimating jointly the destination choice and the trip assignment. Cesario [5] proposed a trip emission-distribution combined model based on zone accessibility. Florian and Nguyen [6] proposed a combined model for simulating jointly distribution, mode choice and assignment. Safwat and Magnanti [7] proposed a model that included all four-stage travel choices (emission, distribution, mode choice and assignment).

The multimodal assignment problem (joint solution of mode choice and assignment problems) was studied by Florian [8], Abdulaal and LeBlanc [9], Dafermos [10], Ortuzar and Willumsen [11], and D'Acierno et al. [12]. Sheffi [13] and Ferrari [14] studied some hyper-network approaches. Southworth and Peterson [15] proposed a multilayer network for simulating intermodal freight transportation.

Simulation of the combined modes was studied among others by Ortuzar [16], Florian and Los [17], Fernandez et al. [18], Friedrich [19], and Garcìa and Marìn [20]. Vovsha [21] proposed the Cross Nested Logit model that allows mode choice to be simulated when combined modes, such as park-and-ride, are available (see also section 3 ).

The outline of the paper is the following: the simulation of intermodal trips is introduced in section 2; the Cross Nested Logit model (Vovsha [21]) is examined in section 3 as well as the equivalent Nested Logit model, which will be used in the proposed model; the hyper-network model is proposed in section 4; section 5 concludes and the mathematical proof is reported in Appendix A.

\section{Simulation of intermodal trips}

Simulation of transportation systems requires implementation of mathematical models that can generally be classified as follows:

- supply models, that represent the transportation facilities, with their connections and performances, and the services (e.g. transit lines with their features);

- demand models, that simulate user travel choices and generate Origin- 
Destination (OD) matrices;

- interaction demand-supply models (or assignment models) that estimate user flows on the network.

A broad description of these models can be found in numerous transportation system engineering books (see, for instance, Cascetta [1]).

Generally, supply models consider road transportation networks and transit networks separately; both are modelled using the well-known graph theory.

Transportation demand is generally modelled assuming that a user has four choice dimensions: choosing whether to move or not, choosing the destination, choosing the mode and choosing the path. These choice dimensions are included in the well-known four-stage demand model system. The first two stages, usually known as generation and distribution (or destination choice) models, generate 'all-mode' OD matrices; the third model (mode choice) generates monomodal OD matrices (such as the car matrix, the mass-transit matrix, the pedestrian matrix and so on). Finally the route choice model estimates the route choice probabilities on each monomodal network.

If demand is assumed rigid, only the route choice probabilities are influenced by congested link costs, while the other choice probabilities are invariable and, therefore, the monomodal OD matrices are constant. Assuming the demand elastic, instead, implies that other choice probabilities depend on congested link costs; from a theoretical point of view, this assumption is always more correct than the other, mainly due to the mode choice dimension that is strongly influenced by network costs.

If there are park-and-ride $(\mathrm{P}+\mathrm{R})$ facilities, they have to be considered as an option among available modes; therefore it is worth considering demand elastic at least at mode choice level and utilising a mode choice model that is able to simulate also $\mathrm{P}+\mathrm{R}$ trips.

In this paper we propose a (supply) hyper-network model that allows us to perform mode choice on a network within route choice and to simulate intermodal trips. Therefore, in following sections we examine the Cross Nested Logit mode choice model (section 3), that also allows us to consider the $\mathrm{P}+\mathrm{R}$ among choice alternatives, and the proposed hyper-network model (section 4).

\section{The cross nested logit model}

For simulating mode choice when park-and-ride is available, Vovsha [21] proposed the Cross Nested Logit (CNL) model. This model considers a choice tree where park-and-ride belongs to two nests (see Figure 1): the road system (RS) nest and the (mass-)transit system (TS) nest.

The choice probabilities can be calculated by the following relations:

-Non-combined trips: $\quad p(\mathrm{C})=p(\mathrm{C} \mid \mathrm{RS}) \cdot p(\mathrm{RS})$

$$
\begin{aligned}
& p(\mathrm{~B})=p(\mathrm{~B} \mid \mathrm{TS}) \cdot p(\mathrm{TS}) \\
& p(\mathrm{M})=p(\mathrm{M} \mid \mathrm{TS}) \cdot p(\mathrm{TS})
\end{aligned}
$$

- Combined trips: $p(\mathrm{P}+\mathrm{R})=p(\mathrm{P}+\mathrm{R} \mid \mathrm{RS}) \cdot p(\mathrm{RS})+p(\mathrm{P}+\mathrm{R} \mid \mathrm{TS}) \cdot p(\mathrm{TS})$ 
In order to obtain a hyper-network model that is able to simulate jointly mode choice, route choice and transfers ( $\mathrm{P}+\mathrm{R}$ trips), we have to transform the $\mathrm{CNL}$ model into a Nested Logit (NL) equivalent model.

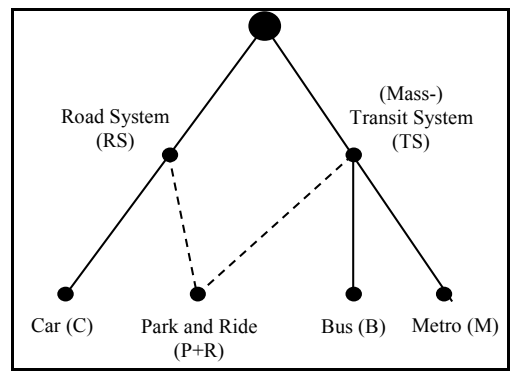

Figure 1: $\quad$ The cross nested logit model.

The choice probability of a combined mode can be obtained as the sum of the choice probabilities of several alternatives, which in the paper we indicate as 'virtual modes'. Obviously, all virtual modes generated by the same combined mode use the same network elements. So in eqn (1) two virtual modes can be identified: $\mathrm{P}+\mathrm{R}^{\mathrm{RS}}$ and $\mathrm{P}+\mathrm{R}^{\mathrm{TS}}$, with the following choice probabilities:

$$
\begin{aligned}
p\left(\mathrm{P}+\mathrm{R}^{\mathrm{RS}}\right) & =p(\mathrm{P}+\mathrm{R} \mid \mathrm{RS}) \cdot p(\mathrm{RS}) \\
p\left(\mathrm{P}+\mathrm{R}^{\mathrm{TS}}\right) & =p(\mathrm{P}+\mathrm{R} \mid \mathrm{TS}) \cdot p(\mathrm{TS})
\end{aligned}
$$

Since in the assignment procedure the flow on a link is the sum of all flows that share the same link, considering $\mathrm{P}+\mathrm{R}$ trips subdivided between two virtual modes does not modify the final results. Therefore, the CNL model (shown in Figure 1) is transformed into the NL model of Figure 2.

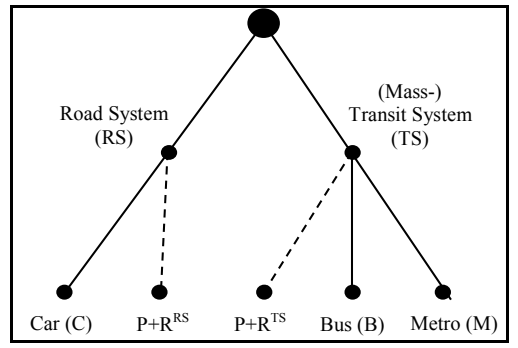

Figure 2: $\quad$ The nested logit equivalent model.

Vovsha [21] proposed to define a level of belonging of $\mathrm{P}+\mathrm{R}$ to $\mathrm{RS}$ and TS that can be obtained by defining a parameter $\alpha_{\mathrm{m}}$ (where $\alpha_{\mathrm{m}} \in[0 ; 1]$ ) that represents the aliquot of $\mathrm{P}+\mathrm{R}$ as belonging to TS (obviously $1-\alpha_{\mathrm{m}}$ is the aliquot of $\mathrm{P}+\mathrm{R}$ as belonging to $\mathrm{RS}$ ).

In this case, the choice probabilities can be expressed as: 


$$
\begin{gathered}
p(\mathrm{C} \mid \mathrm{RS})=\exp \left(V_{\mathrm{C}} / \theta\right) /\left(\exp \left(V_{\mathrm{C}} / \theta\right)+\left(1-\alpha_{m}\right) \cdot \exp \left(V_{\mathrm{P}+\mathrm{R}} / \theta\right)\right) \\
p(\mathrm{P}+\mathrm{R} \mid \mathrm{RS})=\left(1-\alpha_{m}\right) \cdot \exp \left(V_{\mathrm{P}+\mathrm{R}} / \theta\right) /\left(\exp \left(V_{\mathrm{C}} / \theta\right)+\left(1-\alpha_{m}\right) \cdot \exp \left(V_{\mathrm{P}+\mathrm{R}} / \theta\right)\right) \\
p(\mathrm{RS})=\exp \left(V_{\mathrm{RS}} / \theta_{0}\right) /\left(\exp \left(V_{\mathrm{RS}} / \theta_{0}\right)+\exp \left(V_{\mathrm{TS}} / \theta_{0}\right)\right) \\
p(\mathrm{~B} \mid \mathrm{TS})=\exp \left(V_{\mathrm{B}} / \theta\right) /\left(\exp \left(V_{\mathrm{B}} / \theta\right)+\exp \left(V_{\mathrm{M}} / \theta\right)+\alpha_{m} \cdot \exp \left(V_{\mathrm{P}+\mathrm{R}} / \theta\right)\right) \\
p(\mathrm{M} \mid \mathrm{TS})=\exp \left(V_{\mathrm{M}} / \theta\right) /\left(\exp \left(V_{\mathrm{B}} / \theta\right)+\exp \left(V_{\mathrm{M}} / \theta\right)+\alpha_{m} \cdot \exp \left(V_{\mathrm{P}+\mathrm{R}} / \theta\right)\right) \\
p(\mathrm{P}+\mathrm{R} \mid \mathrm{TS})=\alpha_{m} \cdot \exp \left(V_{\mathrm{P}+\mathrm{R}} / \theta\right) /\left(\exp \left(V_{\mathrm{B}} / \theta\right)+\exp \left(V_{\mathrm{M}} / \theta\right)+\alpha_{m} \cdot \exp \left(V_{\mathrm{P}+\mathrm{R}} / \theta\right)\right) \\
p(\mathrm{TS})=\exp \left(V_{\mathrm{TS}} / \theta_{0}\right) /\left(\exp \left(V_{\mathrm{RS}} / \theta_{0}\right)+\exp \left(V_{\mathrm{TS}} / \theta_{0}\right)\right)
\end{gathered}
$$

where: $V_{\mathrm{C}}$ is the systematic utility of car mode; $V_{\mathrm{P}+\mathrm{R}}$ is the systematic utility of park-and-ride mode; $V_{\mathrm{B}}$ is the systematic utility of bus mode; $V_{\mathrm{M}}$ is the systematic utility of metro mode; $V_{\mathrm{TS}}$ is the systematic utility of the mass-transit system; $V_{\mathrm{RS}}$ is the systematic utility of the road system; $\theta$ is the Gumbel parameter associated to the mode choice level $(\mathrm{C}, \mathrm{P}+\mathrm{R}, \mathrm{B}$ and $\mathrm{M}) ; \theta_{0}$ is the Gumbel parameter associated to the system choice level (RS and TS); $\alpha_{m}$ is the parameter representing the aliquot of $\mathrm{P}+\mathrm{R}$ as belonging to TS.

For each Origin-Destination (OD) pair it is necessary to set the value of $\alpha_{m}$. Hence we propose to assume that the user considers the aliquot of $\mathrm{P}+\mathrm{R}$ belonging to TS as the ratio between the sum of transit link costs to the sum of all link costs belonging to the chosen path, $k$ :

$$
a_{m k}=\sum_{l \in T S(k)} c_{l} / C_{k} \quad \text { with: } \quad C_{k}=\sum_{l \in T S(k)} c_{l}+\sum_{l \in R S(k)} c_{l}
$$

where: $\alpha_{m k}$ is the parameter for the intermodal path $k ; l$ represents a link; TS $(k)$ is the set of links that belong to system TS on path $k ; R S(k)$ is the set of links that belong to system RS on path $k ; c_{l}$ is the cost of link $l ; C_{k}$ is the cost of the intermodal path $k$.

Even if other more sophisticated methods can be proposed, in the following we will assume that, for each OD pair, the $\alpha_{m k}$ parameter is equal to the $\alpha_{m k \text {-min }}$, i.e. the $\alpha_{m}$ parameter corresponding to the minimum path, $k$-min. It is thus possible to calculate the $\alpha_{m k}$ parameter by a simple minimum path algorithm on the hyper-network.

\section{The proposed hyper-network model}

Since a $\mathrm{P}+\mathrm{R}$ trip can be seen as belonging to the road system or mass-transit system, we have to assume that a user chooses the system (RS or TS), the mode $\left(\mathrm{C}, \mathrm{P}+\mathrm{R}^{\mathrm{RS}}, \mathrm{P}+\mathrm{R}^{\mathrm{TS}}, \mathrm{B}\right.$ or $\left.\mathrm{M}\right)$ and finally the path $k$, following the choice tree reported in Figure 3. 


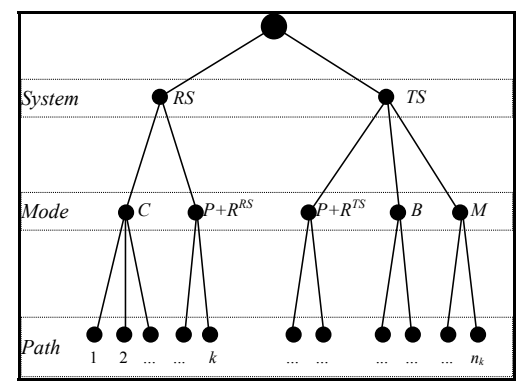

Figure 3: $\quad$ The nested logit choice tree.

For each OD pair the probability $p(s, m, k)$ that a user chooses jointly system $s$, mode $m$ and path $k$, can be obtained as:

$$
p(s, m, k)=p(s) \cdot p(m \mid s) \cdot p(k \mid m, s)
$$

where: $p(s)$ is the choice probability of system $s ; p(m \mid s)$ is the choice probability of mode $m ; p(k \mid m, s)$ is the choice probability of path $k$.

The choice probability of system $s$ can be expressed as:

$$
p(s)=\exp \left(\left(\theta_{1} \cdot Y_{s}+S E_{s}\right) / \theta_{0}\right) / \sum_{s^{\prime} \in I_{s}} \exp \left(\left(\theta_{1} \cdot Y_{s^{\prime}}+S E_{s^{\prime}}\right) / \theta_{0}\right)
$$

where: $\theta_{0}$ is the Gumbel parameter associated to the system choice level (RS and $\mathrm{TS}) ; \theta_{1}$ is the Gumbel parameter associated to the mode choice level $\left(\mathrm{C}, \mathrm{P}+\mathrm{R}^{\mathrm{RS}}\right.$, $\mathrm{P}+\mathrm{R}^{\mathrm{TS}}, \mathrm{B}$ and $\left.\mathrm{M}\right) ; Y_{s}$ is the logsum variable of system $s\left(\theta_{1} \cdot Y_{s}\right.$ is the inclusive utility of system $s$ ); $S E_{s}$ represents the socio-economic attributes relative to system $s$ and the other attributes except the inclusive utility; $s$ ' represents the generic system belonging to the set of available systems $I_{s}$.

The choice probability of mode $m$ can be expressed as:

$$
p(m \mid s)=\frac{\exp \left(\left(\theta_{2} \cdot Y_{m \mid s}+S E_{m \mid s}+\theta_{1} \cdot \ln \gamma_{m \mid s}\right) / \theta_{1}\right)}{\sum_{m^{\prime} \in I_{m \mid s}} \exp \left(\left(\theta_{2} \cdot Y_{m^{\prime} \mid s}+S E_{m^{\prime} \mid s}+\theta_{1} \cdot \ln \gamma_{m^{\prime} \mid s}\right) / \theta_{1}\right)}
$$

where: $\theta_{2}$ is the Gumbel parameter associated to the route choice level; $Y_{m \mid s}$ is the logsum variable of mode $m\left(\theta_{2} \cdot Y_{m / s}\right.$ is the inclusive utility of mode $\left.m\right) ; S E_{m \mid s}$ represents the socio-economic attributes relative to mode $m$ and the other attributes except the inclusive utility; $m$ ' represents the generic mode belonging to the set of available modes $I_{m / s} ; \gamma_{m \mid s}$ is a parameter that depends on the rate of belonging of a mode $m$ to a system $s: \gamma_{m \mid s}=1$ if the mode is 'pure' (i.e. car, bus, metro, etc.); $\gamma_{m \mid s}=\gamma_{m \mid s}\left(\alpha_{m}\right)$ if the mode is combined. For $\mathrm{P}+\mathrm{R}$ modes we propose to use $\gamma_{m \mid s}=\alpha_{m}$ if $s$ is the mass-transit system (TS) and $\gamma_{m \mid s}=\left(1-\alpha_{m}\right)$ if $s$ is the road system (RS).

The choice probability of path $k$ can be expressed as:

$$
p(k \mid m, s)=\exp \left(V_{k \mid m, s} / \theta_{2}\right) / \sum_{k^{\prime} \in I_{k \mid m, s}} \exp \left(V_{k^{\prime} \mid m, s} / \theta_{2}\right)
$$


where: $V_{k \mid m, s}$ is the systematic utility of path $k$, of mode $m$ belonging to system $s$; $k^{\prime}$ represents the generic path belonging to the set of available paths $I_{k \mid m, s}$.

The logsum variables $Y_{s}$ and $Y_{m / s}$ are calculated as:

$$
\begin{gathered}
Y_{m \mid s}=\ln \sum_{k^{\prime} \in I_{k \mid m, s}} \exp \left(V_{k^{\prime} \mid m, s} / \theta_{2}\right) \\
Y_{s}=\ln \sum_{m^{\prime} \in I_{m \mid s}} \exp \left(\left(\theta_{2} \cdot Y_{m^{\prime} \mid s}+S E_{m^{\prime} \mid s}+\theta_{1} \cdot \ln \gamma_{m^{\prime} \mid s}\right) / \theta_{1}\right)
\end{gathered}
$$

Finally, eqn (2) becomes:

$$
\begin{gathered}
p(s, m, k)=\frac{\exp \left(\left(\theta_{1} \cdot Y_{s}+S E_{s}\right) / \theta_{0}\right)}{\sum_{s^{\prime} \in I_{s}} \exp \left(\left(\theta_{1} \cdot Y_{s^{\prime}}+S E_{s^{\prime}}\right) / \theta_{0}\right)} . \\
\frac{\exp \left(\left(\theta_{2} \cdot Y_{m \mid s}+S E_{m \mid s}+\theta_{1} \cdot \ln \gamma_{m \mid s}\right) / \theta_{1}\right)}{\sum_{m^{\prime} \in I_{m \mid s}} \exp \left(\left(\theta_{2} \cdot Y_{m^{\prime} \mid s}+S E_{m^{\prime} \mid s}+\theta_{1} \cdot \ln \gamma_{m^{\prime} \mid s}\right) / \theta_{1}\right)} \cdot \frac{\exp \left(V_{k \mid m, s} / \theta_{2}\right)}{\sum_{k^{\prime} \in I_{k \mid m, s}} \exp \left(V_{k^{\prime} \mid m, s} / \theta_{2}\right)}
\end{gathered}
$$

Eqn (3) represents a Nested Logit model for the choice of system, mode and path. The model (3) can be applied by considering different network models for road and mass-transit systems; in this case the networks are not linked to each other and are independent.

In this paper we propose a hyper-network model that represents with one network the overall multimodal system; to this hyper-network the all-mode OD matrix can be assigned. The assignment procedure also performs the modal split and calculates the flows on transfer links (from road to transit network).

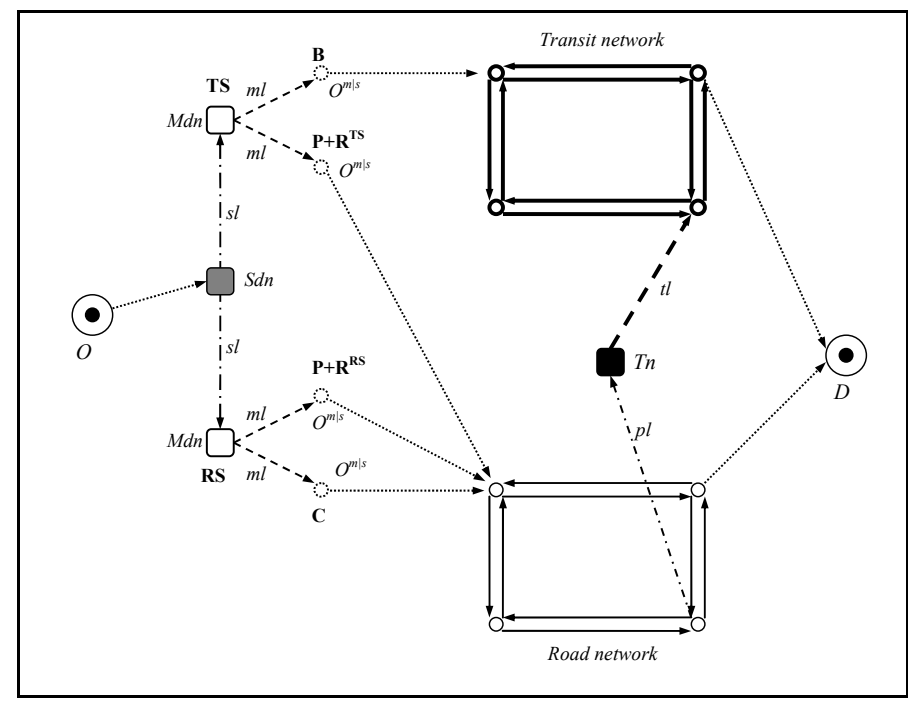

Figure 4: Hyper-network supply model. 
The hyper-network model is reported in Figure 4 and its features are described in the following.

An origin node, $O$, is linked to a system diversion node, $S d n$, that represents a virtual point in which the users choose between the RS and TS; the links that connect $O$ to $S d n$ are named connectors.

A system diversion node, $S d n$, is linked to two (or more, in general) mode diversion nodes, $M d n$, that represent virtual points in which the users choose among the modes that belong to that system; the links that connect $S d n$ to $M d n$ are named system links, $s l$.

A mode diversion node, $M d n$, is linked to more origin mode nodes, $O^{m \mid s}$, one for each mode, $m$, that belongs to the system, $s$; each node is linked to its respective monomodal network by a connector. The links that connect the mode diversion nodes to the origin mode nodes are named mode links, $m l$. Each monomodal network is made up by links and nodes as usually occurs in transportation supply models. Other connectors link some nodes of monomodal networks with destination nodes, $D$.

Some road network nodes are linked to a transfer node, $T n$, with a parking link, $p l$, that represents the cost of parking at a park-and-ride facility (it can also be null); transfer nodes are located where the park-and-ride facilities are sited in the road network. Each transfer node is connected to a transit network node by a transfer link, $t l$, which represents the cost of passing from the road to the transit network (e.g. waiting time, pedestrian time, etc.).

Obviously, the network is built so that for each trip it is not possible to pass through a transfer link more than one time.

For a clearer graphical representation, especially for real dimension networks, it would be better to represent the network in a three-dimensional way, as reported in Figure 5.

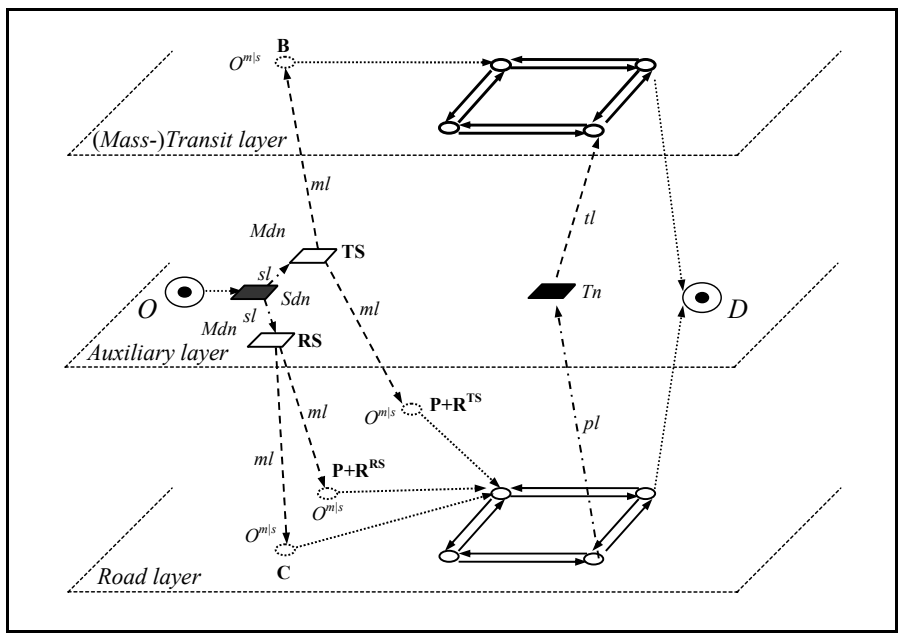

Figure 5: Three-dimensional representation of the hyper-network. 
If $V_{s, m, k}$ is the systematic utility of a path on the hyper-network, adopting a Logit model the path choice probability can be expressed as:

$$
p(s, m, k)=\exp \left(V_{s, m, k} / \theta_{2}\right) / \sum_{s^{\prime} \in I_{s}} \sum_{m^{\prime} \in I_{m s^{\prime}}{ }^{\prime} \in I_{l_{k \mid m^{\prime}, s^{\prime}}}} \exp \left(V_{s^{\prime}, m^{\prime}, k^{\prime}} / \theta_{2}\right)
$$

In Appendix A it is stated that the model (4) is equivalent to the model (3) if on the system links and on the mode links the following cost functions are adopted:

$$
\begin{gathered}
c_{s y s t}^{s}=-\theta_{2} \cdot\left(\left(\theta_{1} / \theta_{0}\right)-1\right) \cdot Y_{s}-\left(\theta_{2} / \theta_{0}\right) \cdot S E_{s} \quad \forall s \in I_{s} \\
c_{m o d}^{m \mid s}=-\theta_{2} \cdot\left(\left(\theta_{2} / \theta_{1}\right)-1\right) \cdot Y_{m \mid s}-\left(\theta_{2} / \theta_{1}\right) \cdot S E_{m \mid s}-\theta_{2} \cdot \ln \gamma_{m \mid s} \quad \forall m \in I_{m \mid s}, \forall s \in I_{s}
\end{gathered}
$$

For proper application of the proposed model it is useful to define several user classes with different characteristics (car availability, parking costs, value of time, etc.); if we indicate user class with apex $c$, the cost functions can be written as:

$$
\begin{gathered}
c_{s y s t}^{s, c}=-\theta_{2} \cdot\left(\left(\theta_{1} / \theta_{0}\right)-1\right) \cdot Y^{c}{ }_{s}-\left(\theta_{2} / \theta_{0}\right) \cdot S E^{c}{ }_{s} \quad \forall s \in I_{s}, \forall c \in I_{c} \\
c_{m o d}^{(m \mid s), c}=-\theta_{2} \cdot\left(\left(\theta_{2} / \theta_{1}\right)-1\right) \cdot Y^{c}{ }_{m \mid s}-\left(\theta_{2} / \theta_{1}\right) \cdot S E^{c}{ }_{m \mid s}-\theta_{2} \cdot \ln \gamma^{c} m \mid s \\
\forall m \in I_{m \mid s}, \forall s \in I_{s}, \forall c \in I_{c}
\end{gathered}
$$

where $I_{c}$ is the set of different classes.

For calculating the flows on the hyper-network it is possible to use a multiuser assignment algorithm.

Anyway, with the proposed approach the route choice model (4) used within an assignment algorithm allows the multimodal equilibrium flows to be calculated on the whole multimodal transportation system, simulating also multimodal trips.

As an assignment model, we propose to use a fixed-point model (according to Cantarella [22]), formally expressed as:

$$
f^{*}=A_{h y p} P_{s, m, k}\left(A_{h y p}{ }^{T} c\left(f^{*}, S E\right)+C^{N A}\right) d^{A M}
$$

where: $\boldsymbol{f}^{*}$ is the multimodal user flow vector: it contains the flows on all links (road, transit, system, mode, parking and transfer) of the hyper-network; $\boldsymbol{A}_{\boldsymbol{h y p}}$ is the link-path incidence matrix of the hyper-network; $\boldsymbol{P}_{\boldsymbol{s}, \boldsymbol{m}, \boldsymbol{k}}$ is the path choice probability matrix; the elements of this matrix are calculated by eqn (4); $c$ is the link cost vector: it contains the costs on all links (road, transit, system, mode, parking and transfer) of the hyper-network; $\boldsymbol{S E}$ is the vector of socio-economic attributes that influence the costs of system and mode links; $C^{\mathrm{NA}}$ is the vector of non-additive path costs, such as waiting times on the transit network; $\boldsymbol{d}^{\boldsymbol{A M}}$ is the vector of all-mode transportation demand.

The existence of a solution to the fixed-point problem (9) is ensured by continuity of function $\boldsymbol{f}$ according to the Brouwer theorem (see Cantarella [22]; Cascetta [1]). Indeed, the elements of matrix $\boldsymbol{P}_{s, m, k}$ are continuous functions (with continuous first partial derivatives) of path costs, since the models used for the path choice have non-null variance, and cost functions are continuous. Also the 
cost functions of mode links and system links are continuous, since the inclusive utilities are continuous with $\boldsymbol{f}$ and the socio-economic attributes are constant.

Sufficient condition for the uniqueness of a fixed-point solution is that the Jacobian of cost functions is a positive definite matrix (see Sheffi and Powell [23]). It is true if elements of matrix $\boldsymbol{P}_{\boldsymbol{s}, \boldsymbol{m}, \boldsymbol{k}}$ are defined by decreasing monotone functions of path costs and elements of the vector $c(f)$ are non-decreasing function of link flows; this last condition is not satisfied for the proposed model if we assume a cross-congestion between transit and road networks: the mode link costs relative to transit systems can decrease with increasing flow if buses do not use exclusive lanes. Indeed, a user increase on the transit system corresponds to a decrease in users on the road system that can produce a reduction in travel costs on both systems.

\section{Conclusions and research prospects}

In this paper a hyper-network model is proposed for simulating multimodal and intermodal (park-and-ride) trips; this model allows the flows of different modes to be calculated jointly under the assumption of elastic demand at mode choice level. In particular, we have stated theoretically the perfect equivalence in terms of user choice probabilities between the proposed approach (based on the hyperpath model) and classical models (sequential implementation of mode and path choice models).

The model has practical applications in planning differentiated parking pricing policies in the centre and in the interchange parking areas, simulating their effects on modal split. Future research could be undertaken to test the model on real networks and develop a decision support system that may help local administrations to test demand management policies.

\section{Appendix A}

In this appendix, we state that adopting cost functions (5) and (6), model (4) is equivalent to model (3). Indeed, the cost of path $s, m, k$ on the hyper-network is given by:

$$
\begin{gathered}
C_{s, m, k}=c_{s y s t}^{s}+c_{m o d}^{m \mid s}+C_{k}^{m \mid s}= \\
=-\theta_{2} \cdot\left(\frac{\theta_{1}}{\theta_{0}}-1\right) \cdot Y_{s}-\frac{\theta_{2}}{\theta_{0}} \cdot S E_{s}-\theta_{2} \cdot\left(\frac{\theta_{2}}{\theta_{1}}-1\right) \cdot Y_{m \mid s}-\frac{\theta_{2}}{\theta_{1}} \cdot S E_{m \mid s}-\theta_{2} \cdot \ln \gamma_{m \mid s}+C_{k}^{m \mid s}
\end{gathered}
$$

where $C_{k}^{m \mid s}$ is the cost of path $k$ of mode $m$ belonging to system $s$.

Since $V_{k \mid m, s}=-C_{k}^{m \mid s}$ and $V_{s, m, k}=-C_{s, m, k}$, it is possible to write:

$$
V_{s, m, k}=-\theta_{2} \cdot\left(\frac{\theta_{1}}{\theta_{0}}-1\right) \cdot Y_{s}-\frac{\theta_{2}}{\theta_{0}} \cdot S E_{s}+
$$




$$
-\theta_{2} \cdot\left(\frac{\theta_{2}}{\theta_{1}}-1\right) \cdot Y_{m \mid s}-\frac{\theta_{2}}{\theta_{1}} \cdot S E_{m \mid s}-\theta_{2} \cdot \ln \gamma_{m \mid s}+V_{k \mid m, s}
$$

Replacing eqn (A.1) into eqn (4), we obtain:

$$
\begin{gathered}
=\exp \left(\frac{p(s, m, k)=}{\theta_{2} \cdot\left(\frac{\theta_{1}}{\theta_{0}}-1\right) \cdot Y_{s}+\frac{\theta_{2}}{\theta_{0}} \cdot S E_{s}+\theta_{2} \cdot\left(\frac{\theta_{2}}{\theta_{1}}-1\right) \cdot Y_{m \mid s}+\frac{\theta_{2}}{\theta_{1}} \cdot S E_{m \mid s}+\theta_{2} \cdot \ln \gamma_{m \mid s}+V_{k \mid m, s}} \theta_{2}\right) . \\
\cdot\left(1 /\left(\sum _ { s ^ { \prime } \in I _ { s } } \sum _ { m ^ { \prime } \in I _ { m | s ^ { \prime } } } \sum _ { k ^ { \prime } \in I _ { k | m ^ { \prime } , s ^ { \prime } } } \operatorname { e x p } \left(\frac { 1 } { \theta _ { 2 } } \cdot \left(\theta_{2} \cdot\left(\frac{\theta_{1}}{\theta_{0}}-1\right) \cdot Y_{s^{\prime}}+\frac{\theta_{2}}{\theta_{0}} \cdot S E_{s^{\prime}}+\theta_{2} \cdot\left(\frac{\theta_{2}}{\theta_{1}}-1\right) \cdot Y_{m^{\prime} \mid s^{\prime}}\right.\right.\right.\right. \\
\left.\left.\left.\left.+\frac{\theta_{2}}{\theta_{1}} \cdot S E_{m^{\prime} \mid s^{\prime}}+\theta_{2} \cdot \ln \gamma_{m^{\prime} \mid s^{\prime}}+V_{k^{\prime} \mid m^{\prime}, s^{\prime}}\right)\right)\right)\right)
\end{gathered}
$$

Since $\exp \left(\left(\theta_{2} \cdot\left(\left(\theta_{1} / \theta_{0}\right)-1\right) \cdot Y_{s^{\prime}}+\left(\theta_{2} / \theta_{0}\right) \cdot S E_{s^{\prime}}\right) / \theta_{2}\right)$ does not depend on $k$ ' and $m^{\prime}$, and $\exp \left(\left(\theta_{2} \cdot\left(\left(\theta_{2} / \theta_{1}\right)-1\right) \cdot Y_{m^{\prime} \mid s^{\prime}}+\left(\theta_{2} / \theta_{1}\right) \cdot S E_{m^{\prime} \mid s^{\prime}}+\theta_{2} \cdot \ln \gamma_{m^{\prime} \mid s^{\prime}}\right) / \theta_{2}\right)$ does not depend on $k^{\prime}$, it is possible to write:

$$
\begin{aligned}
& =\exp \left(\frac{\left(\theta_{1}-\theta_{0}\right) \cdot Y_{s}+S E_{s}}{\theta_{0}}\right) \cdot \exp \left(\frac{\left(\theta_{2}-\theta_{1}\right) \cdot Y_{m \mid s}+S E_{m \mid s}+\theta_{1} \cdot \ln \gamma_{m \mid s}}{\theta_{1}}\right) \cdot \exp \left(\frac{V_{k \mid m, s}}{\theta_{2}}\right) . \\
& \cdot\left(1 /\left(\sum_{s^{\prime} \in I_{s}} \exp \left(\frac{\left(\theta_{1}-\theta_{0}\right) \cdot Y_{s^{\prime}}+S E_{s^{\prime}}}{\theta_{0}}\right)\right. \text {. }\right. \\
& \left.\left.\cdot \sum_{m^{\prime} \in I_{m \mid s^{\prime}}} \exp \left(\frac{\cdot\left(\theta_{2}-\theta_{1}\right) \cdot Y_{m^{\prime} s^{\prime}}+\cdot S E_{m^{\prime} \mid s^{\prime}}+\theta_{1} \cdot \ln \gamma_{m^{\prime} \mid s^{\prime}}}{\theta_{1}}\right) \cdot \sum_{k^{\prime} \in I_{k \mid m^{\prime}, s^{\prime}}} \exp \left(\frac{V_{k^{\prime} \mid m^{\prime}, s^{\prime}}}{\theta_{2}}\right)\right)\right)=
\end{aligned}
$$

Since:
a) $\sum_{k^{\prime} \in I_{k \mid m^{\prime}, s^{\prime}}} \exp \left(V_{k^{\prime} \mid m^{\prime}, s^{\prime}} / \theta_{2}\right)=\exp \left(\ln \left(\sum_{k^{\prime} \in I_{k \mid m^{\prime}, s^{\prime}}} \exp \left(V_{k^{\prime} \mid m^{\prime}, s^{\prime}} / \theta_{2}\right)\right)=\exp \left(Y_{m^{\prime} \mid s^{\prime}}\right)\right.$;
b) $\exp \left(Y_{m \mid s}\right)=\sum_{k^{\prime} \in I_{k \mid m, s}} \exp \left(V_{k^{\prime} \mid m, s} / \theta_{2}\right)$;
c) $\sum_{m^{\prime} \in I_{m \mid s^{\prime}}} \exp \left(\left(\theta_{2} \cdot Y_{m^{\prime} \mid s^{\prime}}+S E_{m^{\prime} \mid s^{\prime}}+\theta_{1} \cdot \ln \gamma_{m^{\prime} \mid s^{\prime}} / \theta_{1}\right)\right)=\exp \left(Y_{s^{\prime}}\right)$;
d) $\exp \left(Y_{s}\right)=\sum_{m^{\prime} \in I_{m \mid s}} \exp \left(\left(\theta_{2} \cdot Y_{m^{\prime} \mid s}+S E_{m^{\prime} \mid s}+\theta_{1} \cdot \ln \gamma_{m^{\prime} \mid s}\right) / \theta_{1}\right)$;

we obtain: 


$$
\begin{gathered}
=\frac{\exp \left(\left(\theta_{1} \cdot Y_{s}+S E_{s}\right) / \theta_{0}\right)}{\sum_{s^{\prime} \in I_{s}} \exp \left(\left(\theta_{1} \cdot Y_{s^{\prime}}+S E_{s^{\prime}}\right) / \theta_{0}\right)} \cdot \\
\frac{\exp \left(\left(\theta_{2} \cdot Y_{m \mid s}+S E_{m \mid s}+\theta_{1} \cdot \ln \gamma_{m \mid s}\right) / \theta_{1}\right)}{\sum_{m^{\prime} \in I_{m \mid s}} \exp \left(\left(\theta_{2} \cdot Y_{m^{\prime} \mid s}+S E_{m^{\prime} \mid s}+\theta_{1} \cdot \ln \gamma_{m^{\prime} \mid s}\right) / \theta_{1}\right)} \cdot \frac{\exp \left(V_{k \mid m, s} / \theta_{2}\right)}{\sum_{k^{\prime} \in I_{k \mid m, s}} \exp \left(V_{k^{\prime} \mid m, s} / \theta_{2}\right)}
\end{gathered}
$$

that is eqn (3).

\section{References}

[1] Cascetta E., Transportation systems analysis: models and applications, Springer: New York (NY), USA, 2009.

[2] Tomlin, J.A., A mathematical programming model for the combined distribution-assignment of traffic, Transportation Science, 5(2), pp. 122140, 1971.

[3] Florian, M., Nguyen, S. \& Ferland, J., On the combined distributionassignment of traffic, Transportation Science, 9(1), pp. 43-53, 1975.

[4] Evans, S.P. Derivation and analysis of some models for combining trip distribution and assignment, Transportation Research, 10(1), pp. 37-57, 1976.

[5] Cesario, F.J., A combined trip generation and distribution model, Transportation Science, 9(3), pp. 211-223, 1975.

[6] Florian, M. \& Nguyen, S., A combined trip distribution, modal split and trip assignment model, Transportation Research, 12(4), pp. 241-246, 1978.

[7] Safwat, K.N.A. \& Magnanti, T.L., A combined trip generation, trip distribution, modal split, and trip assignment model, Transportation Science, 22(1), pp. 14-30, 1988.

[8] Florian, M., A traffic equilibrium model of travel by car and public transit modes, Transportation Science, 11(2), pp. 166-179, 1977.

[9] Abdulaal, M. \& LeBlanc, L.J., Methods for combining modal split and equilibrium assignment models, Transportation Science, 13(4), pp. 292 314, 1979.

[10] Dafermos, S.C., The general multimodal network equilibrium problem with elastic demand, Networks, 12(1), pp. 57-72, 1982.

[11] Ortuzar, J. de D. \& Willumsen, L.G., Modelling transport, John Wiley \& Sons, Chichester, United Kingdom, 1994.

[12] D’Acierno, L., Montella, B. \& Gallo, M., Multimodal assignment to congested networks: fixed-point models and algorithms, Proc. of the European Transport Conference 2002, Cambridge, United Kingdom, 2002.

[13] Sheffi, Y., Urban transportation networks, Prentice-Hall, Englewood Cliffs (NJ), USA, 1985.

[14] Ferrari, P., Capacity constraints in urban transport networks, Transportation Research Part B, 31(4), pp. 291-301, 1997 
[15] Southworth, F. \& Peterson, B.E., Intermodal and international freight network modeling, Transportation Research Part C, 8(1-6), pp. 147-166, 2000.

[16] Ortuzar, J. de D., Nested logit models for mixed-mode travel in urban corridors, Transportation Research Part A, 17(4), pp. 283-299, 1983.

[17] Florian, M. \& Los, M., Determining intermediate origin-destination matrices for the analysis of composite mode trips, Transportation Research Part B, 13(2), pp. 91-103, 1979.

[18] Fernandez, E., De Cea, J., Florian, M. \& Cabrera, E., Network equilibrium models with combined modes, Transportation Science, 28(3), pp. 182-192, 1994.

[19] Friedrich, M., A multi-modal transport model for integrated planning. World transport research: Volume 2 - Planning, operation, management and control, eds. H. Meersman, E. Van de Voorde \& W. Winkelmans, Pergamon Press, Oxford, United Kingdom, pp. 1-14, 1999.

[20] Garcì, R. \& Marìn, A., Network equilibrium with combined modes: model and solution algorithms, Transportation Research Part B, 39(3), pp. 223254, 2005.

[21] Vovsha, P., Application of cross-nested logit model to mode choice in Tel Aviv, Israel, metropolitan area, Transportation Research Record, 1607, pp. 6-15, 1997.

[22] Cantarella, G.E., A general fixed-point approach to multimode multi-user equilibrium assignment with elastic demand, Transportation Science, 31(2), pp. 107-128, 1997.

[23] Sheffi, Y. \& Powell, W., A comparison of stochastic and deterministic traffic assignment over congested networks, Transportation Research Part $B$, 15(1), pp. 53-64, 1981. 